\title{
Regular pairs for solving Fredholm integral equations of the second kind
}

\author{
ALEJANDRA ORELLANA CASTILLO, SILVIA SEMINARA ${ }^{\dagger}$ AND MARÍA INÉS \\ TROPAREVSKY
}

\author{
Date of Receiving : $\quad 30.03 .2018$ \\ Date of Revision : 22.08 .2018 \\ Date of Acceptance : 23.08 .2018
}

\begin{abstract}
Classical Fredholm equations of the second kind are integral equations that appear in different areas of applied mathematics, sometimes as equivalent formulation of boundary value problems for ordinary differential equations. Numerical solutions to these equations have been often developed in the context of Galerkin type schemes. In particular, Petrov-Galerkin method has been proposed to solve this type of integral equation by projecting on appropriate finite dimensional subspaces. Recently, the notion of regular pair was introduced. It guarantees solvability and numerical stability of the approximation scheme. Different characterizations of the property of being regular are presented in the literature. In the case of Hilbert spaces, it is related to the positive definitiveness of a matrix. In this work we present pairs of simple subspaces $\left\{X_{n}, Y_{n}\right\}$ that are regular $\forall n \in \mathbb{N}$ and show the goodness of the approximation in two numerical examples.
\end{abstract}

\section{Introduction}

Classical Fredholm equations of the second kind are integral equations of the form

$$
u(t)-\int_{a}^{b} k(s, t) u(s) d s=g(t), \quad t \in[a, b]
$$

where $u$ is the unknown function in a Banach space $X$; the kernel $k:[a, b] \times[a, b] \rightarrow \mathbb{R}$ and the right-hand side $g:[a, b] \rightarrow \mathbb{R}$ are given functions.

These equations usually appear as equivalent formulation of value problems for ordinary differential equations, and are named after Erik Ivar Fredholm (1866-1927) for his numerous contributions to the field.

If we introduce the operator $K: X \rightarrow X$,

$$
K u(t)=\int_{a}^{b} k(s, t) u(s) d s
$$

Key words and phrases. Fredholm integral equations, Numerical methods, Petrov-Galerkin method, Regular pairs.

Communicated by: Ricardo Oscar Sirne

${ }^{\dagger}$ Corresponding author. 